\title{
Refractory Rhabdoid Tumor
}

National Cancer Institute

\section{Source}

National Cancer Institute. Refractory Rhabdoid Tumor. NCI Thesaurus. Code C142858.

Rhabdoid tumor that does not respond to treatment. 